\title{
LAS POLÍTICAS PÚBLICAS DE EVALUACIÓN Y ACREDITACIÓN DE LAS CARRERAS DE GRADO EN ARGENTINA
}

\section{Ariadna Guaglianone ${ }^{1}$}

\section{RESUMEN}

El trabajo hace referencia a los procesos que se desarrollaron en la universidad en Argentina, en las últimas décadas, con la aplicación de las nuevas políticas públicas impulsadas desde el Estado con el objetivo de mejorar la calidad de los programas y las instituciones. Indaga sobre la reforma producida en los años 80 y 90 , en la instalación de las prácticas de evaluación y acreditación de las carreras de grado y en su impacto luego de 15 años de implementación de las mismas. Se considera necesaria la rediscusión de las políticas universitarias para una comprensión más profunda de las características y estilos organizacionales de la universidad argentina.

Palabras clave: educación superior, universidades, políticas públicas de evaluación y acreditación

\section{PUBLIC POLICIES ON EVALUATION AND ACCREDITATION OF CAREERS IN ARGENTINA}

\section{ABSTRACT}

The work makes reference to processes conducted by Argentine universities during the last decades through the implementation of the new public policies encouraged by the Government with the purpose of improving quality of programs and institutions. The work also addresses reforms introduced during the eighties and nineties in the evaluation and accreditation processes for the undergraduate programs, and their impact fifteen years after their implementation. To understand more deeply the features and organizational types of Argentine universities, further analysis of the university policies is required.

Keywords: higher education, universities public policies of evaluation and accreditation

1 Universidad Abierta Interamericana (UAI) y Facultad Latinoamericana de Ciencias Sociales (FLACSO).Argentina. Contacto: gualianone8@hotmail.com 


\section{LAS POLÍTICAS PÚBLICAS DE EVALUACIÓN Y ACREDITACIÓN DE LAS CARRERAS DE GRADO EN ARGENTINA}

\section{La educación superior en Argentina}

La universidad argentina fue, en el contexto latinoamericano, una de las primeras instituciones creadas bajo el modelo napoleónico de universidad. El movimiento de reforma universitaria iniciado en Córdoba en 1918, le imprimió características distintivas expresadas, fundamentalmente, en una nueva forma de gobierno ${ }^{2}$ mediante el cogobierno del cual comenzaron a participar los profesores, desplazando el poder de elección que tenían las academias externas. La participación de los estudiantes fue entendida como una participación indirecta, desde la elección de los profesores que integrarían los cuerpos colegiados. También le imprimió, a la universidad, un protagonismo político y social que la articulará de forma decidida al campo político, una reivindicación de autonomía frente al Estado y simultáneamente una articulación con las aspiraciones de movilidad social de los sectores medios. Asimismo, impulsó el modelo napoleónico, con una fuerte impronta profesionalista, en contraposición al modelo humboldtiano centrado en la investigación científica y al modelo basado en los intereses de la Iglesia y las clases altas de la sociedad. El movimiento de reforma acentuó el carácter profesionalista de la universidad, centrado en el Estado, los negocios y las profesiones: características propias de la universidad latinoamericana tradicional.

Por otra parte, a lo largo de su historia podemos encontrar largos periodos de represión política ${ }^{3}$, una marcada expansión y

2 El sistema de gobierno, hasta el momento, fijado en los estatutos de 1883 otorgaban el control de las facultades a graduados que formaban parte de las academias. Estos integraban los cuerpos colegiados, se autorreclutaban, eran vitalicios y designaban a sus propios miembros.

3 La interrupción del sistema democrático en Argentina durante los años 1930, 1943, 1955, 1966 y 1976 significó para las universidades, con distintos grados de intensidad, represión, intervención, expulsión de docentes e investigadores, la desintegración de equipos de investigación altamente calificados, entre otras. 
diversificación en los años sesenta y setenta similar al resto de los países latinoamericanos. Sin embargo, sus tradicionales estructuras académicas se mantuvieron en el tiempo resistiendo, adaptándose, refuncionalizando o rechazando las demandas de nuevas reformas y de modernización académica.

En este contexto se puede hablar de una universidad decididamente mesocrática, orientada y moldeada por la demanda de movilidad social, cuya eficiencia interna y externa presenta debilidades difíciles de resolver en una realidad de gobierno universitario que asume la forma de república parlamentaria que dificulta la inducción de cambios desde la cumbre de la institución o el sistema. Por otro lado, la marcada orientación profesionalista de las carreras como soporte del sistema y la importante articulación con el campo político y corporativo profesional debilitan sin duda la capacidad de cambio e innovación desde su base disciplinaria. (Krotsch, 2005).

En los años 80 y 90 el concepto de reforma en la educación superior pone el eje en el debate sobre la evaluación y la calidad. Ahora encontramos una sociedad que critica a la universidad y una universidad que debe rendir cuentas a diferentes sectores de la sociedad. El contexto de la reforma en Argentina, durante la década del 80, se asienta sobre el proceso de normalización de la vida universitaria luego de ocho años de dictadura militar y la crisis presupuestaria, con un sistema de educación superior centrado en la tensión entre una progresiva demanda por la misma y una creciente restricción al financiamiento público de las universidades. También, un importante proceso de diversificación de las instituciones producto del gran crecimiento de la demanda y el surgimiento de una variedad de programas académicos vinculados a los estudios de posgrado, junto a la aparición de nuevas modalidades educativas como la enseñanza semipresencial y a distancia.

La masividad, generada por las demandas sociales sobre los sistemas educativos en condiciones de restricción presupuestaria, sumada a la creciente "partidización" y politización de las instituciones, a la falta de financiamiento y al aislamiento del Estado, la sociedad y 
el rechazo al mercado produjeron una crisis de calidad, de eficiencia y de eficacia en la educación superior (Mollis, 1994).

\section{La reforma de la educación superior en la década del 90}

En la década del 90, la agenda internacional junto con los organismos nacionales establecieron tres líneas de política centrales: 1) adopción de formas sistemáticas de evaluación de los programas e instituciones; 2) innovación en cuanto a las formas de asignación del presupuesto público y a las fuentes de financiamiento (recuperación de costos con equidad y competencia por el financiamiento) y 3) inscripción de estas y otras estrategias dentro de marcos normativos consensuados que permitan, a su vez, la formulación de políticas públicas de reforma (Castro J., 2003).

Bajo estas circunstancias, el Estado crea en 1995 el Programa para la Reforma de la Educación Superior (PRES), cofinanciado por el Banco Mundial (BM) ${ }^{4}$. Este programa se desarrolla en el contexto de la recientemente creada Secretaría de Políticas Universitarias (SPU) del Ministerio de Educación, Ciencia y Tecnología (MECyT) y sus objetivos centrales fueron los siguientes: reforma y ordenamiento del marco legal de la educación superior; introducción de incentivos para el mejoramiento de la calidad de la educación terciaria y de la asignación de recursos; desarrollo de las políticas de evaluación y acreditación por medio de la creación de la Comisión Nacional de Evaluación y Acreditación Universitaria (CONEAU)5; mayor transparencia en la gestión mediante el mejoramiento de la información; mejoramiento de la inversión en equipamiento e infraestructura para las universidades públicas; introducción de modificaciones a la distribución de recursos presupuestarios y

4 El Programa de Reforma de la Educación Superior (PRES) fue el producto de un préstamo suscripto entre la República Argentina y el Banco Mundial/Banco Internacional de Reconstrucción y Fomento (BM/BIRF, Préstamo 3921-AR), promulgado por Decreto del Poder Ejecutivo Nacional No 840/95 en 1995. El Programa contó con una inversión de 273 millones de dólares, de los cuales 165 millones fueron financiados por el BIRF.

5 Organismo creado a partir de la sanción de la Ley de Educación Superior № 24.521. La CONEAU es el ente encargado de la evaluación y acreditación de las instituciones universitarias, las carreras de grado y posgrado en Argentina. 
fortalecimiento de la capacidad de conducción y programación de la SPU (Carlino y Mollis, 1997).

A partir de la aplicación de estas nuevas políticas públicas, el Estado pasará a ejercer un tipo de control de carácter indirecto y mediado por los organismos intermedios o de amortiguación: la Secretaría de Políticas Universitarias (SPU), la Comisión Nacional de Evaluación y Acreditación Universitaria (CONEAU) y el Consejo de Universidades ${ }^{6}(\mathrm{CU})$, entre otras. Por los programas especiales: la Comisión de Acreditación de Posgrado (CAP), el Fondo para el Mejoramiento de la Calidad Universitaria (FOMEC), el Sistema de Información Universitaria (SIU) y el Programa de Incentivos a los Docentes Investigadores, que le permitieron vincularse con las universidades nacionales y en algunos casos con las universidades privadas con el objetivo de mejorar la calidad de los programas y de las instituciones.

Estas iniciativas generaron fuertes controversias con gran parte de las universidades nacionales y en menor medida con las universidades privadas. Podemos decir que dos grandes actores jugaron roles preponderantes en la discusión y el debate de las reformas en la educación superior. Por un lado el Consejo Interuniversitario Nacional $^{7}$ (CIN) y por el otro el Ministerio de Educación, Ciencia y Tecnología (MECyT). Asimismo, en el seno del CIN se encontraban posturas diferentes debido a su conformación, integrado por el total de los rectores de las universidades nacionales. En contraposición, el MECyT exhibía una postura compacta que se modificará parcialmente en el juego de las negociaciones con el CIN.

Mientras en el CIN se debatía cómo debería ser la evaluación de las instituciones, el MECyT desarrollaba una política basada

6 El Consejo de Universidades es el organismo que reúne a los rectores de todas las universidades del país. El Consejo se encarga de definir políticas y estrategias de desarrollo universitario, promover la cooperación entre instituciones universitarias, la adopción de pautas para la coordinación del sistema universitario y acordar con el Consejo Federal de Cultura y Educación criterios y pautas para la articulación entre las instituciones educativas de nivel superior.

7 El Consejo Interuniversitario Nacional es un organismo integrado por todos los rectores de las universidades nacionales de Argentina. 
en acuerdos bilaterales con algunas universidades para realizar la evaluación mediante la firma de convenios entre la Secretaría de Políticas Universitarias y cada universidad. Esta situación impidió que el CIN asumiera la coordinación e implementación de un sistema alternativo de evaluación.

En cuanto a la resistencia desde las universidades privadas, representadas en el Consejo de Rectores de Universidades Privadas ${ }^{8}$ (CRUP), la misma se centraba en la oposición a la creación de un ente estatal encargado de la evaluación y propusieron que las instituciones universitarias constituyeran sistemas voluntarios de evaluación y acreditación externa, alternativa que no prosperó.

En tanto, el sector estudiantil se hizo presente en marchas y asambleas. Si bien se manifestaron en contra de la futura Ley de Educación Superior, el tema de la evaluación y acreditación no formó parte de las propuestas concretas que pudo haber realizado este sector.

Para el año 1994 las políticas implementadas desde el MECyT avanzaban hacia la institucionalización de la evaluación de la calidad en el contexto de las concepciones esgrimidas por el ministerio que finalizaron plasmadas, después de arduas negociaciones con el CIN, en la Ley de Educación Superior No 24.521.

A pesar de la diversidad de actores que participaron en el debate y la compleja trama que se fue conformando a partir de las distintas posiciones sobre el tema, las universidades terminaron adaptándose y comprometiéndose con la implementación de estas nuevas políticas.

\section{Las nuevas políticas públicas de evaluación y acreditación}

La creciente complejidad de los sistemas de educación superior vinculada a su masividad, al importante crecimiento del sector privado, sus limitaciones para adaptarse a la nueva sociedad de la información y el conocimiento, llevó al gobierno a abandonar

8 El Consejo de Rectores de Universidades Privadas es el organismo que agrupa a los rectores de las universidades privadas en Argentina. 
la posición de libre mercado propia de la década de los 80, en la que la privatización era considerada un paliativo a la expansión cuantitativa, sin que se controlase la calidad. También, se adoptó una nueva posición mediante la formulación de políticas centradas en el mejoramiento y el aseguramiento de la calidad por medio de la acreditación, la certificación y la evaluación, con el objetivo de conciliar la expansión y la calidad, elaborando instrumentos para su control (Del Bello, 1993).

La reforma producida en los años 90 en Argentina introdujo los conceptos de eficiencia, eficacia, calidad, evaluación y acreditación. Asimismo, informó la necesidad de incorporar cambios sustantivos en la lógica institucional de las universidades (Mignone, 1995).

Frente a esta situación, y antes de impulsar la reforma, el Ministerio de Cultura y Educación (MCE) elabora un diagnóstico sobre el funcionamiento de las instituciones universitarias. Entre las falencias pueden destacarse: la ausencia de un "sistema"; la expansión indiscriminada de la matrícula especialmente en las carreras profesionales; la baja calidad de la formación; la distribución del presupuesto de manera inercial y negociado; una profunda crisis estructural con relación a la proporción de ingresantes-egresados, deserción, altas tasas de repitencia o baja dedicación al estudio; planes de estudio excesivamente largos y no adecuados a las demandas del medio, a los cambios tecnológicos y del mercado; baja dedicación de los profesores tanto a la docencia como a la investigación; insuficiente formación de posgrado en el cuerpo docente; alta tasa de deserción y un régimen de permanencia donde predomina el estudiante crónico.

El MCE, entre 1995 y 1998, toma distintas medidas de política pública para subsanar los problemas detectados en el diagnóstico.

En el proceso de formulación de estas políticas se pueden distinguir tres etapas: la primera (1991-1992) caracterizada por la definición del problema e instalación del tema en el país; la segunda (1993-julio 1995) por la instalación del tema en la agenda universitaria y por la formulación de alternativas de solución; y la tercera (desde julio de 1995) por la institucionalización de la evaluación y la 
acreditación de la calidad universitaria como adopción de una política concreta plasmada en la Ley de Educación Superior No 24.521 y en el Decreto 499/95 (Marquis, 1996).

En el año 1993 el entonces Ministerio de Cultura y Educación de la Nación firmó dieciséis convenios con universidades nacionales y dos con asociaciones de facultades para implementar procesos de evaluación institucional. Estos acuerdos preveían el asesoramiento para la realización de autoevaluaciones, que quedaron en manos de las mismas instituciones y la asistencia para constituir y coordinar comités de evaluación externa. La CONEAU, una vez conformada, toma bajo su responsabilidad la prosecución de los restantes convenios en lo relativo a las evaluaciones externas.

En el año 1994 se crea e implementa el Programa de Incentivos a los docentes-investigadores. Su objetivo fue promover las actividades de investigación en el cuerpo docente. El sistema partió de una categorización de los profesores en cuatro clases, privilegiando la producción científica de los docentes y sus publicaciones en revistas con arbitraje.

También, a finales de 1994, el Ministerio creó la Comisión de Acreditación de Posgrados (CAP). La tarea realizada por la CAP fue la primera experiencia en acreditación de posgrado sistemática y generalizada realizada en el país y constituyó la prueba piloto para la posterior institucionalización de los procesos de acreditación desarrollados por la CONEAU.

En el año 1995 se crea, mediante el decreto presidencial 480/95, el Fondo para el Mejoramiento de la Calidad Universitaria (FOMEC), orientado en un primer momento a apoyar los proyectos de reforma que las universidades decidieran emprender en el contexto del mejoramiento de la calidad de la enseñanza. El FOMEC asignó fondos a las universidades estatales mediante proyectos concursables con cinco convocatorias anuales entre 1995 y $1999^{9}$.

9 El presupuesto asignado al FOMEC era de 230 millones de pesos, de los cuales 145 provenían del Programa del Banco Mundial y los 93 millones restantes correspondían a la contrapartida que debían asegurar las universidades nacionales que participaban en los proyectos. 
El FOMEC financió tres tipos de proyectos: disciplinarios, de biblioteca y de desarrollo institucional. Estos podían solicitar fondos en cuatro rubros: bienes, becas y pasantías, servicios de consultoría y obras de refacciones de carácter menor (Castro, 2003).

La acreditación de los posgrados realizada por la CAP se constituyó en una condición necesaria para que los mismos accedieran, según la categoría obtenida, al financiamiento de mejoras mediante fondos que asignaba el FOMEC.

En síntesis, la reforma procuraba la diferenciación de las instituciones, la diversificación de las fuentes de financiamiento, la redefinición del rol del Estado respecto de la educación superior y la introducción de políticas que asignaran prioridad a los objetivos de calidad y de equidad.

La diversificación institucional se expresó en la expansión del sistema tanto en el ámbito público como en el privado. En este sentido, entre 1989 y 1995 se autorizó el funcionamiento de 22 universidades privadas y se crearon 8 universidades nacionales: 6 de ellas funcionan en el conurbano bonaerense, provincia de Buenos Aires (García de Fanelli, 1997) y 2 surgirán de la nacionalización de instituciones provinciales.

Hasta el año 2010, el sistema universitario argentino registra 40 universidades públicas (sin contar las diferentes sedes de la Universidad Tecnológica Nacional y las numerosas sedes, en otras localidades, de varias universidades nacionales), 7 institutos universitarios públicos, 45 universidades privadas, 12 institutos universitarios privados, 1 universidad provincial, 1 universidad extranjera y 1 universidad internacional. En resumen, existen un total de 107 establecimientos universitarios que poseen una oferta nominal de servicios sobredimensionada y una calidad real del conjunto en permanente cuestionamiento.

También, se produjo la expansión de instituciones de carácter terciario no universitarias junto con la diversificación de su oferta orientada a la atención del sector servicios (informática, diseño, turismo, hotelería). Podría afirmarse que se trataba de instituciones 
caracterizadas por una oferta académica de perfil y de calidad muy heterogénea.

Por otra parte, y por iniciativa de la SPU, se establece como otra vía fundamental de diversificación de la oferta la expansión de las instituciones terciarias no universitarias de formación técnica y profesional. Asimismo, se estimula la creación de los colegios universitarios, los cuales deben ser acreditados por una universidad y ofrecen carreras cortas, flexibles y/o a término ${ }^{10}$.

Con relación a la diversificación de fuentes de financiamiento, para el caso de las instituciones públicas, se innovó mediante la posibilidad de venta de servicios y transferencia de conocimientos al sector privado y la autorización para cobro de arancel, o sea, el arancelamiento de los estudios. Si bien se avanzó en la venta de servicios y transferencia por conocimientos, el cobro de arancel en los estudios de grado no se convirtió en una práctica generalizada, solo se estableció en algunos casos mediante la figura del bono contribución, en las universidades públicas.

Por último, una de las bases centrales de la reforma fue la sanción, en agosto de 1995, de la Ley de Educación Superior $\mathrm{N}^{\circ} 24.521$ (LES), que permitió el surgimiento de un instrumento normativo que regularía el funcionamiento de la educación terciaria en su conjunto. Entre sus principales componentes encontramos que abarca todos los aspectos de la educación superior, comprendiendo tanto las instituciones públicas y privadas como también al sector no universitario; establece la delimitación de responsabilidades en cuanto al sector de dependencia: sector no universitario de responsabilidad jurisdiccional, sector universitario de responsabilidad del Estado Nacional; establece un sistema de evaluación institucional para la educación superior no universitaria y de evaluación y acreditación para la educación superior universitaria; determina competencias

10 El artículo 22 de la Ley de Educación Superior sostiene que "Las instituciones de nivel superior no universitario que se creen o transformen, o las jurisdicciones a las que ellas pertenezcan, que acuerden con una o más universidades del país mecanismos de acreditación de sus carreras o programas de formación y capacitación, podrán denominarse colegios universitarios". 
en el otorgamiento de títulos, asignando como de competencia exclusiva de las universidades el otorgamiento de títulos de grado de licenciatura, maestría y doctorados y fija pautas para el financiamiento de la educación.

Las universidades argentinas, con un fuerte arraigo en las antiguas concepciones de autonomía, no tomaron la iniciativa para solucionar los problemas vinculados con la calidad y la eficiencia del sistema, lo que provocó que las reformas de la educación superior hayan provenido del exterior y no del interior de las mismas. Tanto los sistemas de evaluación como de acreditación han sido producto de políticas estatales y no de propuestas elaboradas por las instituciones universitarias.

La Ley de Educación Superior avanzó en la conformación del sistema de educación superior, dotándolo de entidad propia y entendiéndolo como un conjunto de elementos que tienen cierta unidad, atribuida básicamente al dinamismo de la cooperación y en la que no está ausente el conflicto.

\section{La evaluación institucional y la acreditación en Argentina}

En el contexto de la Ley de Educación Superior se crea la Comisión Nacional de Evaluación y Acreditación Universitaria (CONEAU), organismo descentralizado que funciona en jurisdicción del Ministerio de Educación de la Nación. La creación de la CONEAU significó la incorporación de procedimientos sistemáticos de evaluación y de acreditación en los sistemas nacionales, implicó una redistribución de las relaciones de poder entre el Estado, las elites académicas, sus instituciones y la ejercida por los mercados.

La CONEAU está constituida por doce miembros de reconocida jerarquía académica y científica, con experiencia en la gestión universitaria. Ejercen sus funciones a título personal, con independencia de criterio y sin asumir la representación de ninguna institución. Los miembros de la CONEAU son designados por el poder ejecutivo nacional a propuesta de diversos organismos; el Consejo 
Interuniversitario Nacional propone tres comisionados, al igual que la Cámara de Diputados y el Senado. Por su parte, el Consejo de Rectores de Universidades Privadas, la Academia Nacional de Educación y el Ministerio de Educación, propone cada uno a un comisionado.

Los miembros de la CONEAU son designados por cuatro años, con renovación parcial cada dos. Su presidencia y vicepresidencia son ejercidas por un año, por dos de sus miembros elegidos por mayoría.

Las funciones de la CONEAU se encuentran establecidas en el artículo 46 de la ley, siendo las mismas las siguientes:

a) Coordinar y llevar adelante la evaluación externa prevista en el artículo $44^{11}$;

b) Acreditar las carreras de grado referidas en el artículo $43^{12}$, así como las carreras de posgrado, cualquiera sea el ámbito en que se desarrollen, conforme a los estándares que establezca el Ministerio de Cultura y Educación en consulta con el Consejo de Universidades;

c) Pronunciarse sobre la consistencia y viabilidad del proyecto institucional que se requiere para que el Ministerio de Cultura y Educación autorice la puesta en marcha de una nueva institución universitaria con posterioridad a su creación o el reconocimiento de una institución universitaria provincial;

d) Preparar los informes requeridos para otorgar la autorización provisoria y el reconocimiento definitivo de universidades privadas, así como los informes sobre la base de los cuales se evaluará el periodo de funcionamiento provisorio de dichas instituciones.

11 Las instituciones universitarias deberán asegurar el funcionamiento de instancias internas de evaluación institucional, que tendrán por objeto analizar los logros y dificultades en el cumplimiento de sus funciones, así como sugerir medidas para su mejoramiento. Las autoevaluaciones se complementarán con evaluaciones externas, que se harán como mínimo cada 6 años, en el marco de los objetivos definidos por cada institución. Abarcará las funciones de docencia, investigación y extensión, y en el caso de las instituciones universitarias nacionales, también la gestión institucional. Las evaluaciones externas estarán a cargo de la Comisión Nacional de Evaluación y Acreditación Universitaria o de entidades privadas constituidas con ese fin, conforme se prevé en el artículo 45, en ambos casos con la participación de pares académicos de reconocida competencia. Las recomendaciones para el mejoramiento institucional que surjan de las evaluaciones tendrán carácter público.

$12 \mathrm{El}$ artículo 43 hace referencia a las carreras consideradas de interés público que conllevan un riesgo social y cuyo ejercicio profesional puede comprometer el derecho y la seguridad de las personas. 
La CONEAU se transformó en el único organismo público nacional de evaluación y de acreditación universitaria y comenzó a funcionar en 1996. Sin embargo, la Ley de Educación Superior (LES) autoriza la creación de entidades privadas de evaluación y acreditación (EPEAUs), reconocidas por el ME previo dictamen de la CONEAU. Las entidades privadas pueden cumplir funciones similares a la CONEAU, excepto en lo que respecta a los dictámenes requeridos para la puesta en marcha de universidades nacionales, el reconocimiento de instituciones universitarias provinciales o la autorización de instituciones universitarias privadas.

La misión institucional de la CONEAU se centró en asegurar y mejorar la calidad de las carreras e instituciones universitarias que operan en el sistema universitario argentino por medio de actividades de evaluación y acreditación de la calidad de la educación universitaria.

\section{La evaluación institucional}

Según la CONEAU, "...la evaluación institucional supone un proceso complejo para determinar el valor de algo, que implica una delicada tarea de interpretación de un conjunto de elementos que interactúan configurando una realidad particular y significativa...". "Una evaluación, asimismo, no son los 'datos', aunque estos sean indispensables, sino el proceso por el que se aprecia y discierne el valor de las acciones y realizaciones; un proceso profundamente humano que se nutre y se articula en el diálogo, la discusión y la reflexión".

La evaluación institucional debería contemplar el contexto actual y la historia de la institución, permitir una adecuada relación entre lo particular (unidades académicas) y lo global (la universidad), sin perder de vista que su objetivo es la institución en su conjunto, entendiendo que la misma tiene una identidad que no se conforma como la suma de sus partes. Asimismo, la evaluación institucional debería asumir la diversidad como punto de partida y como orientación principal, no solo entre universidades sino hacia el interior de cada una de ellas. Finalmente, la evaluación institucional necesariamente debería aportar información. Es indudable que la información es una 
base imprescindible para comprender la realidad institucional, así como para formular propuestas de mejora, cambio o conservación.

La evaluación institucional debería ser útil fundamentalmente a la propia universidad evaluada y a la comunidad en general. En concreto, se trata de mejorar la calidad de esta institución mediante el análisis de los resultados, y de forma especial de las acciones, identificando problemas y comprendiéndolos en su contexto.

La evaluación institucional contempla dos fases: la autoevaluación y la evaluación externa, cada una de ellas con diferentes actores.

El informe de autoevaluación consiste en una presentación cuantitativa y cualitativa donde se exponen las actividades, la organización y el funcionamiento de la institución, así como sus objetivos, políticas y estrategias. Constituye un análisis de los procesos y de los resultados obtenidos, así como también una apreciación sobre su realidad actual a partir de su "sociogénesis". Se desarrolla sobre una lógica emergente de la institución, de su proyecto institucional y quienes la realizan son los propios protagonistas de la misma.

En la evaluación externa se aprecia la organización y el funcionamiento de la institución, se observa la trama de su desarrollo, se valoran los procesos y los resultados y se recomiendan cursos de acción. La realizan pares académicos, que no pertenecen a la institución universitaria evaluada, sobre la base del proyecto institucional de la misma y a partir de la autoevaluación realizada.

Las evaluaciones externas valoran, mediante los comités de pares, las funciones de docencia, investigación y extensión y, en el caso de instituciones nacionales, la gestión institucional. El resultado del proceso implica la elaboración de un informe final por parte de dicho comité en donde se explicitan medidas de mejoramiento de la calidad y recomendaciones públicas acerca de la calidad institucional ${ }^{13}$.

13 Los informes de evaluación institucional de las instituciones universitarias solo presentan propuestas de mejoras que las instituciones pueden o no realizar, ya que no existen consecuencias punitivas, por parte del organismo evaluador, sobre la implementación o no de los procesos de mejoramiento. 
La evaluación institucional debe realizarse como mínimo cada seis años y producir recomendaciones para el mejoramiento de las instituciones, cuyo carácter es público (Lineamientos para la Evaluación Institucional, 1997, CONEAU).

\section{Acreditación de las carreras de grado}

La CONEAU tiene entre sus funciones la acreditación periódica de las carreras de grado cuyos títulos corresponden a profesiones reguladas por el Estado y cuyo ejercicio pudiera comprometer el interés público poniendo en riesgo de modo directo la salud, la seguridad, los derechos, los bienes o la formación de los habitantes. Este proceso se basa en la aplicación de estándares que establece el Ministerio de Educación en consulta con el Consejo de Universidades ${ }^{14}$.

Los procedimientos consisten en un informe de autoevaluación realizado por la carrera en donde se describe y analiza su situación actual, valora el nivel de cumplimiento de los estándares establecidos y sobre la base del análisis formula planes de mejoramiento.

La evaluación es realizada por comités de pares constituidos por la CONEAU a partir de las recomendaciones de las respectivas comisiones asesoras basadas en las propuestas de instituciones universitarias y no universitarias.

El resultado del proceso es un dictamen en donde los pares evaluadores juzgan si la carrera cumple con los estándares establecidos y estiman la factibilidad de los planes de mejoramiento. La propuesta final de dictamen realiza una recomendación cuyos resultados posibles son: a) acreditación por seis años (cumplen con el perfil previsto); b) acreditación por tres años (cumplen con el perfil previsto pero no tiene un ciclo completo de dictado); c) acreditación por tres años (no cumplen con el perfil previsto pero tiene estrategias de mejoramiento adecuadas que se transforman en compromisos) y, finalmente, d) la

14 Los estándares para la acreditación de las carreras de grado son elaborados por las asociaciones profesionales y/o las agrupaciones de decanos de cada disciplina. En el caso de la acreditación de posgrado existe la Resolución Ministerial 1168/07 aprobada por el Ministerio de Educación. 
no acreditación (no cumplen con el perfil previsto y sus estrategias de mejoramiento no permiten esperar que se alcancen en un plazo razonable).

Con respecto de los procesos de internacionalización de la educación superior, la CONEAU participa desde el año 2002 en el mecanismo experimental de acreditación (MEXA), mediante el cual se llevan a cabo acreditaciones conjuntas de carrera de grado en los países del MERCOSUR, Bolivia y Chile.

La acreditación se realiza de acuerdo con parámetros de calidad comunes, previamente definidos para el MERCOSUR en el documento "Dimensiones, Componentes, Criterios e Indicadores". Hasta el momento se establecieron de manera experimental la acreditación de las carreras de agronomía, ingeniería y medicina.

\section{Impacto de los procesos de evaluación y acreditación de las carreras de grado}

En Argentina, en los años 90, el Estado tuvo un importante protagonismo en la construcción de las prácticas de evaluación y acreditación universitaria. Su intervención se centró en la producción de mejoras en términos de calidad institucional vinculadas al desarrollo de políticas públicas en el ámbito de la educación superior.

La práctica de la evaluación surgió de un proceso de conflicto y negociación entre los distintos actores del sistema universitario y extrauniversitario. Este conflicto se agudizó por la importancia histórica del sector universitario y por la carencia de políticas públicas estables en el tiempo, implementadas desde el Estado para la educación superior.

Pese a ello, la práctica de la acreditación respondió más a un consenso y a un acuerdo con la comunidad académica que aceptaron las políticas de aseguramiento de la calidad.

La participación de las asociaciones profesionales y de las agrupaciones de decanos en la elaboración y discusión de los estándares permitió involucrar a la comunidad académica en los 
procesos de acreditación de las carreras de grado de un modo más participativo. La discusión generó consensos en la necesidad de llevar adelante procesos de mejora en la calidad de las carreras.

En un principio, la integración del sistema de educación superior estuvo focalizada en el Estado como instrumento que detentaba el poder de integrar. Podría hablarse de una situación histórico-política en donde el poder del gobierno era más fuerte que el de las instituciones y, por ende, las mismas poseían menores posibilidades de negociación. Las universidades se encontraban en un contexto de normalización, crisis presupuestaria y escasez de fondos para la investigación, traducida en una pronunciada dependencia del gobierno. Es necesario destacar que en el momento de desarrollo de las políticas públicas enmarcadas en el Programa de Reforma de la Educación Superior (PRES), el gobierno contaba con una inversión del Banco Internacional de Reconstrucción y Fomento (BIRF).

También, existía una pérdida de poder en los ámbitos de gestión de las universidades que repercutió en un espacio menor para la negociación frente a un Estado con más poder y en el contexto de una mayor complejidad del sistema de educación superior, no solo por la variedad de instituciones, sino, además, por la existencia de múltiples organismos de coordinación y toma de decisiones.

Posteriormente, una vez avanzada la aplicación de las políticas en la coordinación del sistema ${ }^{15}$, se produce un giro al eje Estadooligarquía académica, en donde la última asumió un rol significativo al transformarse en parte de los denominados "cuerpos intermedios de amortiguación” (Clark, 1992), que pasaron a regular las relaciones entre el Estado y las universidades, como la Comisión Nacional de Evaluación y Acreditación Universitaria (CONEAU), entre otros.

15 Burton Clark desarrolla distintos procesos de integración distinguiendo la coordinación burocrática, la coordinación política y la coordinación de mercado. Estos procesos de integración estarían dando cuenta del cambio organizacional y serían una consecuencia de la modificación en las modalidades de coordinación del sistema. Las modificaciones pueden girar en torno al Estado (por medio de modalidades políticas o burocráticas), el mercado (mercado de consumidores, mercado ocupacional y mercado de reputaciones institucionales) y las "oligarquías profesionales". A partir de estos tres tipos ideales de coordinación, Clark elabora un modelo triangular, teniendo en cuenta los casos de estudio, donde cada vértice supone el predominio de una forma de coordinación sobre las otras dos. 
Además, se establece una burocracia funcional al interior de las instituciones vinculada a los nuevos procesos de evaluación y de acreditación fomentando desde adentro la institucionalización de las prácticas. Se crean sectores que se benefician con las nuevas políticas y son los funcionarios quienes llevarán adelante las mismas en el espacio de las universidades. Son los "técnicos en evaluación y acreditación", los "expertos o pares evaluadores" que permiten incorporar a los procesos a una gran parte de la oligarquía académica.

Esta comunidad, creada a partir de la figura de expertos o pares evaluadores, adquieren ciertas características vinculadas con: 1) ser beneficiarios de ciertos privilegios, como la libertad de investigar y de enseñar; 2) ser objeto de una heterogeneidad que atraviesa su tarea, dada por la tensión entre una pertenencia disciplinar versus una pertenencia institucional y 3) poseer cierta tendencia al conservadurismo dada por una estructura de regulaciones que protege los intereses legítimos de los académicos (Clark, 1992).

La oligarquía académica comienza a operar en el espacio de la evaluación, en permanente tensión y confrontación con diversos intereses, elaborando juicios evaluativos cruzados con intereses particulares vinculados con intereses individuales y a su pertenencia disciplinar o institucional.

A pesar del predominio del eje Estado-oligarquía académica se observan elementos que suponen una orientación hacia el mercado y la academia. En el primer caso se distingue mediante la desregulación que se produce en materia de planes de estudio y contenidos, en los programas de financiamiento estatal según indicadores de calidad y eficiencia, en la creación de entes privados de evaluación y acreditación y en una mayor competencia entre las instituciones por alumnos, profesores, reputaciones y recursos. En el segundo caso, en la lógica académica, se observa en el fuerte hincapié sobre la investigación y la carrera académica.

Desde su inicio, los procesos de evaluación y acreditación, han desarrollado un carácter político, como espacios sociales de disputa de valores y de poder, además de técnico y metodológico, que se expresa en negociaciones, acuerdos y conflictos entre los actores 
involucrados. Podemos decir que el conjunto de teorías, conceptos y prácticas vinculadas a la evaluación y la acreditación constituyen y conforman un campo en donde se ponen en juego valores e intereses que posicionan a los actores en distintos lugares respecto de los bienes materiales y simbólicos en disputa. Es en este campo y entre sus actores (gobierno, académicos, empresarios, gestores institucionales, estudiantes, entre otros) donde se define la agenda de la evaluación y la acreditación, los medios para implementarlas y los logros obtenidos.

La evaluación se desarrolla en un contexto político, en el sentido de que las acciones institucionales y las carreras que se evalúan son producto de la toma de decisiones políticas. Asimismo, la evaluación al llevarse a cabo para colaborar en la toma de decisiones entra inevitablemente en la arena política y la evaluación en sí misma adopta una postura política debido a que, por su naturaleza, expone explícitamente cuestiones vinculadas con las problemáticas, debilidades, legitimidades de objetivos y estrategias de carreras e instituciones.

Las políticas de evaluación no significaron cambios sustantivos en la base del sistema. Fueron apropiadas por las universidades porque resultaban convenientes para el logro de determinados objetivos. En algunos casos, la evaluación institucional permitió avanzar en procesos de cambio que la universidad no puede llevar adelante sola, por la creciente conflictividad interna que generan estos, sin el aval de una mirada externa. En otros casos permitió orientar la gestión y dar una direccionalidad a políticas consensuadas. También, las prácticas de evaluación fueron utilizadas como herramientas para acompañar y dar impulso a los proyectos decididos por la propia universidad.

La evaluación permitió cambios en la medida que generó procesos antes inexistentes, como la autoevaluación integral de la institución, pero no replanteos significativos sobre la visión, misión y desarrollo de la institución universitaria. La evaluación tuvo un impacto de mejoramiento mediante el ordenamiento de los datos, la elaboración de estadísticas y en algunos casos la constitución de oficinas o dependencias encargadas del seguimiento e implementación de los procesos y en otros en el desarrollo de planes estratégicos. Del 
mismo modo, logró movilizar a los distintos actores de la comunidad académica, aunque muy acotadamente, ante la necesidad de contacto entre las distintas áreas y sectores.

Se considera que la mirada externa generó una combinación entre cierta reflexibilidad frente a estos procesos y una adaptación formal vinculada con la legalidad de los mismos.

Los procesos de evaluación, en general, solo abarcan a un sector de la comunidad académica, que es el que participa en la gestión o por un interés particular, aunque en gran medida parecería ser más por obligación que por la existencia de una conciencia sobre la importancia del proceso.

Podemos decir que las universidades en la década del 90, en un principio, resistieron las políticas públicas que se impulsaban desde el gobierno, pero al mismo tiempo se adaptaron a las mismas mediante sus acciones.

En Argentina, las condiciones institucionales para el desarrollo de los procesos de evaluación institucional han dependido en gran medida de la existencia de dos factores: un impulso externo, en este caso la Ley de Educación Superior junto con la existencia de un organismo que implementa las políticas de evaluación pero que armoniza con las necesidades de la gestión, y un liderazgo fuerte que maneja las tensiones y negociaciones que surgen de estos procesos.

La escasa existencia, hasta el momento, de una conciencia de la importancia de estos procesos para el mejoramiento de las universidades en todos los actores que conforman la comunidad académica de las instituciones, la ausencia de financiamiento para la implementación de las mejoras que surgen en los informes de evaluación realizados por los pares y la burocratización de los mismos, tanto por el organismo de aplicación como de las instituciones, ha creado solo una incipiente "cultura de la evaluación" en las universidades, impidiendo incorporar dicho proceso como una instancia de autodiagnóstico permanente que permita a la misma revisar sus objetivos y metas orientados al mejoramiento de sus prácticas académicas. 
Se observa que las evaluaciones institucionales no han alcanzado a generar procesos de reforma y cambio con el mismo dinamismo que la acreditación de las carreras.

La experiencia acumulada hasta el momento en los procesos de evaluación institucional ha demostrado que los mismos fueron producto de acuerdos establecidos entre las instituciones y la CONEAU, permitiendo formular un diagnóstico de las instituciones evaluadas y una serie de mejoras vinculadas a los aspectos organizativos formales más que a cambios sustantivos al interior de las instituciones. Esta situación estaría vinculada a la ausencia de consecuencias expresas una vez recibidos los informes de evaluación de los pares y a la inexistencia de mecanismos de financiamiento para la implementación de las mejoras.

Las consecuencias de la acreditación de las carreras de grado, vinculadas a la posibilidad del cierre de una carrera, generaron una solución intermedia en la etapa de implementación del proceso por el cual una carrera puede acreditar por un tiempo menor con compromisos de mejoramiento. De esta manera, una política pensada y basada en el aseguramiento de la calidad se transformó en una política para el mejoramiento de las mismas.

La práctica de la acreditación de las carreras de grado ha sido considerada por la comunidad académica como una experiencia positiva, en donde se han producido procesos de aprendizaje y de concientización sobre el estado de las carreras y las facultades, sobre las fortalezas y las debilidades de las mismas. Igualmente, al existir compromisos expresos que demandan un tiempo establecido con el organismo evaluador se han efectuado resultados concretos vinculados con la rendición de cuentas de los avances en los procesos de mejoramiento asumidos por las carreras.

Se considera que los procesos de acreditación de las carreras de grado tuvieron una mayor aceptación que los procesos de evaluación institucional dentro de la comunidad académica. Esta situación se debe a que dicha comunidad participa activamente en el proceso. Es la propia comunidad científica la que determina y acuerda los estándares con los cuales se acreditarán las carreras. Estos procesos 
de discusión y de acuerdos garantizan la participación y la inclusión en las problemáticas que se consideran relevantes para la formación de los graduados y una mayor conciencia en el ámbito de las carreras sobre la necesidad de llevar adelante estos procesos.

La acreditación de las carreras de grado ha permitido la articulación horizontal del sistema con relación a las carreras que se van sumando a las prácticas de acreditación con la generación de acuerdos entre distintas instituciones universitarias que han pasado por dichos procesos. Estos acuerdos permiten tanto la integración del sistema en su conjunto como la movilidad de alumnos entre las carreras de una misma zona y el reconocimiento de tramos curriculares. Asimismo, se observa en la comunidad de académicos un proceso de consolidación interno enmarcado en un sentimiento de pertenencia a una carrera y a una unidad académica que se diferencia del resto a raíz de haber experimentado el proceso de acreditación.

La acreditación de las carreras de grado produjo una situación inversa con relación a la evaluación institucional. La acreditación aceleró los procesos de cambio en las carreras a partir de la necesidad de cumplir con el estándar, se convirtió en una herramienta para la planificación y la gestión en las instituciones. Estos procesos de mejora estarían vinculados con tres cuestiones: el carácter punitivo del proceso, la continuidad obligatoria de la acreditación (la mayoría de las carreras han acreditado por 3 años con compromisos para cumplir que son revisados por los pares evaluadores) y la posibilidad de acceso a financiamiento para el cumplimiento de los compromisos.

Se considera, como un efecto no deseado, que la práctica de la acreditación de las carreras de grado ha producido un proceso de absorción de la evaluación institucional. Las instituciones universitarias y sus organismos de gestión se encuentran más comprometidos en los procesos de acreditación que de evaluación institucional.

Teniendo en cuenta este proceso es posible afirmar que la acreditación de las carreras de grado ha empezado a generar, al interior de las universidades, en especial en las nacionales, una diferenciación sustantiva entre las carreras que se someten a los procesos de acreditación y las que no se encuentran sujetas a dichos procesos. 
Esta diferenciación se plasma no solo en una atención particular que se genera en la unidad académica, sino también en la acción de la universidad como contraparte necesaria para el acceso a los fondos que financian los procesos de mejoramiento.

La existencia de fondos competitivos a los que acceden las carreras acreditadas para financiar sus mejoras y compromisos ha comenzado a generar disparidades en términos de "mejoramiento de la calidad" en las carreras comprometidas en el artículo $42^{16} \mathrm{y}$ que no requieren procesos de acreditación como son las ciencias sociales y humanidades donde las políticas públicas parecerían no estar diseñadas para estimular o crear espacios de calidad en este ámbito, sino para acentuar su menor calidad en términos académicos e institucionales frente a las ciencias básicas y tecnológicas.

También, se observa que la diferenciación ocurre entre las carreras del área disciplinaria de las ciencias básicas y de las ciencias sociales y humanidades. Mientras que en el primer caso las instituciones se encuentran abocadas a cumplir los compromisos de las carreras acreditadas y financiar los proyectos de mejora, en el segundo caso las carreras continúan profundizando sus dificultades e incrementando sus debilidades.

Cabría preguntarse si, como impacto no deseado, la acreditación de las carreras de grado no estaría generando, por un lado, una diferenciación entre las mismas. Carreras, motivo de reflexión de los académicos de la propia comunidad y de los expertos evaluadores, que además permiten la detección de las fortalezas y debilidades y la consecución de planes de mejoras financiadas tanto por la propia universidad en el caso de las instituciones privadas como por la universidad y el Estado en el caso de las instituciones públicas. Por el otro lado, se trata de ver a las carreras que hasta el momento no han

16 Artículo 42: Los títulos con reconocimiento oficial certificarán la formación académica recibida y habilitarán para el ejercicio profesional respectivo en todo el territorio nacional, sin perjuicio del poder de policía sobre las profesiones que corresponde a las provincias. Los conocimientos y capacidades que tales títulos certifican, así como las actividades para las que tienen competencia sus poseedores, serán fijados y dados a conocer por las instituciones universitarias, debiendo los respectivos planes de estudio respetar la carga horaria mínima que para ello fije el Ministerio de Cultura y Educación, en acuerdo con el Consejo de Universidades. 
sido evaluadas; que tienen un cuerpo docente con bajas dedicaciones, serios problemas de infraestructura, que no acceden a recursos presupuestarios adicionales pero que, paradójicamente, son las que generan la principal masa de egresados del país.

Este proceso de diferenciación que se va desarrollando entre las distintas unidades académicas, según acrediten o no sus carreras, pone en cuestión la idea de la universidad como un todo. A la vez, parecería difícil que los procesos de evaluación institucional, que fueron el comienzo de las políticas públicas de mejoramiento de las instituciones universitarias, pudieran desarrollarse a la par de los procesos de acreditación teniendo en cuenta que la evaluación institucional necesita de la articulación de las unidades académicas, que aún no ha sido resuelto el eje del financiamiento de las mejoras y que requiere de una movilización que englobe, en su conjunto, a la comunidad académica de la institución.

La acreditación de las carreras de grado estaría derivando en una diferenciación tan importante dentro de cada institución y entre las distintas unidades académicas que la componen que habría producido el efecto de que la mayoría de las carreras quieran ser incorporadas en el artículo 43 de la Ley de Educación Superior y transitar los procesos de acreditación en busca de calidad, reconocimiento, prestigio y recursos.

Frente a estos nuevos desafíos podría pensarse, por un lado, en la revisión de cuáles deberían ser las carreras incluidas en el artículo 43 poniendo un límite a su inclusión y, por otro, un mecanismo que acredite las carreras comprendidas en el artículo 42, que podría ser voluntario debido al carácter de la mismas, pero que les permita a las instituciones una reflexión interna sobre las debilidades y fortalezas para la elaboración de propuestas de mejoramiento. El establecimiento de una política de "contrato-programa"17 podría solucionar el financiamiento de las mejoras.

17 En la Argentina el Ministerio de Educación, Ciencia y Tecnología impulsó durante el año 2005 la implementación de una política de contratos-programa que utilizaba como diagnóstico las evaluaciones institucionales y que servirán para financiar los proyectos institucionales de las universidades. Debido a problemas financieros no se avanzó en la implementación de los mismos. 
Un problema creciente que se observa en los procesos de evaluación y acreditación se vincula con el trabajo realizado por los comités de pares evaluadores que han generado algunos conflictos con las instituciones y las carreras relacionadas por las interpretaciones subjetivas que dificultan aplicaciones homogéneas para casos similares. Paralelamente, se observa la ausencia de un reconocimiento de la diversidad institucional existente evaluando al conjunto del sector universitario con los patrones de la universidad pública a pesar de su franco deterioro en varias de sus unidades académicas, de su baja tasa de graduación, de sus altas tasas de deserción, de la ausencia de políticas de investigación, de concursos docentes y de gestiones basadas más en políticas partidarias que académicas. Esta situación se advierte tanto en las posturas del organismo evaluador como de los informes de los comités de pares evaluadores.

En los procesos de acreditación de las carreras de grado se observa una tendencia a otorgar un mayor peso a ciertas actividades por sobre otras. Esta situación podría tergiversar el sentido del artículo 43, establecimiento de estándares sobre competencias profesionales que resguarden a la sociedad, teniendo en cuenta que los académicos responden maximizando sus objetivos de elevar su ingreso y su prestigio volcándose a funciones que, de acuerdo con instancias varias de evaluación y categorización, otorgan mayor puntaje. Teniendo en cuenta los instrumentos utilizados para la acreditación y la ponderación que los pares evaluadores hacen de su lectura parecería más conveniente escribir artículos cortos para publicarlos en revistas con arbitraje y asistir a conferencias internacionales prestigiosas, que destinar tiempo a la docencia de grado o a proyectos de investigación de largo plazo (García de Fanelli, 2000).

En este sentido quien no se adecue a dichos patrones, aun cuando, por ejemplo, cumpla con eficiencia y calidad con la función de formar buenos profesionales, se verá obligado a tratar de parecer lo que realmente no es, pudiendo incluso perjudicar la labor que en términos aceptables venía realizando hasta el presente (García de Fanelli, 2000).

La evaluación y acreditación como regulación y aseguramiento de la calidad se tradujo en prácticas con supuesta objetividad 
apoyadas en instrumentos que pretenden convertir a los procesos evaluativos en búsquedas de datos o indicadores mensurables y a los evaluadores en el contexto de una estricta neutralidad. Al respecto, Neave (2001) habla de una "instrumentalidad abrumadora" tendiente a inducir a los académicos a cumplir con los objetivos, las metas y los fines de los dirigentes. Esta visión del "cómo" evaluar se diferencia de concebir a la evaluación como "un proceso amplio de conocimiento, interpretación, atribución de juicios de valor, organización e instauración de acciones y metas para el mejoramiento y el cumplimiento de las finalidades públicas y sociales de las instituciones" (Díaz Sobrinho, 2003: 38).

Una cuestión que debería ser eje de discusiones entre los actores del sistema es la real efectividad de los procesos de evaluación institucional y de la acreditación de carreras de grado, vinculados con los usos que el Estado realiza de ambos procesos.

En el caso de la evaluación institucional de las universidades no parece haber un uso de la misma por parte del Estado como insumo para el diseño y desarrollo de políticas sectoriales, ni para la distribución presupuestaria, ni para gestionar el funcionamiento del "mercado universitario".

Hasta el momento, pareciera que solamente el énfasis de las políticas de Estado con relación a los informes producidos por la CONEAU, está orientado a mejorar las carreras de grado acreditadas. Esto se refleja mediante los programas de financiamiento de la Secretaría de Políticas Universitarias formulados a tal efecto (PROMEI, PROMFYB, PROMVET, PROMARQ y PROMAGRO ${ }^{18}$ ).

Mientras que respecto de los informes de evaluación institucional no existen programas específicos tendientes a alcanzar las transformaciones necesarias.

18 PROMEI, PROMFYB, PROMVET, PROMARQ y PROMAGRO son programas que financian los proyectos de mejora de las universidades nacionales surgidas de los procesos de acreditación de las carreras de grado de ingeniería, farmacia y bioquímica, veterinaria, arquitectura y agronomía. 
Finalmente, no hay duda de que tanto los procesos de evaluación institucional como de acreditación de las carreras de grado devinieron en mejoras sustantivas para el conjunto de las instituciones universitarias. Entre ellas se destaca el ordenamiento de los datos, la elaboración de estadísticas, la comunicación entre las distintas áreas, la concientización de los problemas de las instituciones y de las carreras y, en la mayoría de los casos, la posibilidad de desarrollar planes de mejora.

No obstante, aún quedan cuestiones por definir vinculadas con el financiamiento de las mejoras, sobre todo en los procesos de evaluación institucional, la mirada de los pares respecto de la aplicación de los estándares, el desgaste que surge de los procesos constantes de acreditación, la participación de los actores universitarios, la conciencia relacionada al seguimiento de estos procesos como parte de una actividad permanente en las instituciones, la articulación al interior de las instituciones de ambos procesos y, por sobre todo, la diferenciación que está surgiendo, en el caso de las universidades nacionales, entre las carreras definidas como de interés público y que por ende ingresan al proceso de acreditación y aquellas que no se consideran para los procesos de acreditación, entre otras.

Siguiendo este razonamiento, deberíamos preguntarnos si la implementación de las políticas de evaluación institucional y de acreditación de las carreras de grado, producto de importantes debates y controversias dentro del sistema de educación superior, no requiere de un proceso de retroalimentación de las políticas públicas que permita articular una gama de resultados comunes de dichos procesos que podrían estar señalando debilidades más propias del sistema universitario que de cada una de las universidades, a fin de diseñar nuevas estrategias que permitan su superación.

\section{A modo de conclusión}

Las políticas en educación superior desarrolladas a partir de la década del 90 produjeron un cambio y un viraje en la política universitaria argentina hacia la incorporación creciente de la calidad y el mejoramiento de las carreras e instituciones como centro de las políticas y la gestión. 
La implementación de las políticas llevadas adelante por la instancia de coordinación creada por la Ley de Educación Superior, la Comisión Nacional de Evaluación y Acreditación Universitaria (CONEAU), se concretó mediante algunas experiencias aisladas de autoevaluación institucional, a las cuales siguieron procesos de evaluación externa y por medio de la institucionalización de la evaluación de la calidad y de los procesos de acreditación de programas de posgrado y grado.

Es oportuno señalar como un logro importante desde la implementación de las políticas de evaluación y de acreditación el desarrollo de una incipiente "cultura de la evaluación", vinculada con la aceptación de dichas prácticas por los actores del sistema.

Sin embargo, existen algunas cuestiones que aún generan importantes escenarios de incertidumbre en las políticas de educación superior. La principal cuestión residiría en una evaluación de la calidad y la efectividad de los procesos implementados, más allá de la incipiente "cultura de la evaluación", del mejoramiento de los sistemas de ordenamiento de datos y de la aceptación de las políticas de evaluación institucional y acreditación de carreras de grado y posgrado en la comunidad universitaria.

Después de aproximadamente quince años de implementación tanto de las políticas de evaluación institucional como de acreditación estamos frente a la necesidad de discutir nuevamente las políticas universitarias para una comprensión más profunda de las características y estilos organizacionales de la universidad argentina. Se observa que muchas de las políticas dirigidas a la universidad se fundan en diagnósticos generales que no tienen en cuenta la particularidad de un sistema integrado por distintos tipos de instituciones. Esta situación ha impedido reconocer las culturas organizacionales que orientan la práctica de los actores. Esta visión lineal y acotada ha imposibilitado observar la complejidad de las instituciones, redundando en reformas educativas formales que ponen en duda la efectividad de los procesos de evaluación y acreditación. 


\section{Referencias bibliográficas}

Castro, J. (2003). "Fondos competitivos y cambio académico e institucional en las universidades públicas argentinas. El caso del Fondo para el Mejoramiento de la Calidad Universitaria (FOMEC)". Documento de Trabajo (110), pp. 1-16. Buenos Aires, Universidad de Belgrano.

Clark, B. (1992). El sistema de educación superior. México, Editorial Nueva Imagen.

Comisión Nacional de Evaluación y Acreditación Universitaria (1997), Lineamientos para la Evaluación Institucional. Buenos Aires. Argentina.

Del Bello, J. C. (1993). "Relaciones entre el Estado y la Universidad". Pensamiento Universitario. Año 1 (1), pp. 41- 45. Buenos Aires. Argentina.

Diaz Sobrinho, J. (2003) "Avaliacão da Educacão Superior. Regulacão e emancipacão", en RAIES, vol. 8, n. ${ }^{\circ} 2$.

García de Fanelli, A. M. (1997) "Las nuevas universidades de conurbano bonaerense: misión, demanda externa y construcción de un mercado académico". Documento CEDES (117). Buenos Aires, Serie Educación Superior.

García de Fanelli, A. M. (2000). "Transformaciones en la política de educación superior Argentina en los años noventa". Revista de la Educación Superior en Linea (114). Buenos Aires, Argentina.

Krotsch, P. (2005). La evaluación de la calidad en la Argentina: la necesidad de un análisis centrado en el poder y el conflicto. Buenos Aires, Instituto de Investigaciones Gino Germani. Universidad de Buenos Aires.

Marquis, C. (1996). Conferencia de la UNESCO, Citado en Sánchez Martínez, Eduardo. "La evaluación de las instituciones universitarias, Estado de la cuestión”. Universidades. Gestión y Evaluación de la calidad universitaria. Argentina. Unión Industrial Argentina.

Mignone, E. (1995). "Educación en los 90: el desafío de la calidad, la pertinencia, la eficacia y la equidad". Boletín de la Academia Nacional de Educación (19), pp. 4 -15. Argentina. Academia Nacional de Educación.

Mollis, M. (1994). Crisis, calidad y evaluación de las universidades: tres temas para el debate. En: Krotsch, P., Puiggros A. (Comp.). Universidades y Evaluación: estado de la discusión. Buenos Aires. Editorial Aike.

Neave, G. (2001). Educación Superior: historia y política. Estudios comparativos sobre la universidad contemporánea. Gedisa. 
Pugliese, J. C. (2003). Políticas de Estado para la Universidad Argentina. Balance de una gestión en el nuevo contexto nacional e internacional. Buenos Aires. Argentina.

\section{Fuentes electrónicas}

http://www.coneau.edu.ar

http://www.cin.edu.ar

http://www.me.gov.ar/spu

Ley de Educación Superior No 21.521.

Consejo Interuniversitario Nacional (CIN). 1992. Acuerdo Plenario No 50. Evaluación de la calidad universitaria.

Consejo Interuniversitario Nacional (CIN). 1993. Acuerdo Plenario No 97. Informe final del Subproyecto 06.

Consejo Interuniversitario Nacional (CIN). 1993. Acuerdo Plenario No 98. Evaluación y mejoramiento de la calidad.

Consejo Interuniversitario Nacional (CIN). 1994. Acuerdo Plenario Nº 133. Documento básico de evaluación de la calidad.

Consejo Interuniversitario Nacional (CIN). 1994. Acuerdo Plenario Nº 140. Proyecto de Ley de Educación Superior.

Consejo Interuniversitario Nacional (CIN). 1994. Acuerdo Plenario No 144. Ley de Educación Superior.

INFOMEC: Boletín informativo del Fondo para el Mejoramiento de la Calidad Universitaria (1996). Ministerio de Cultura y Educación. Buenos Aires, Argentina.

\section{Bibliografía recomendada}

Carlino, F.; Mollis M. (1997). "Políticas internacionales, gubernamentales e interinstitucionales de evaluación universitaria. Del Banco Mundial al CIN". Revista del Instituto de Investigaciones en Ciencias de la Educación. Año VI, (10), pp. 22-36. Buenos Aires, Miño y Dávila Editores.

Fernández, N. (2003). "Evaluación y Acreditación en la Educación Superior Argentina". La Evaluación y la Acreditación de la Educación Superior en América Latina y el Caribe, pp. 3-33. IESAL/UNESCO. IES/2003/ED/ $\mathrm{PI} / \mathrm{IO}$.

García de Fanelli, A. M. (1997). "Las nuevas universidades de conurbano bonaerense: misión, demanda externa y construcción de un mercado académico". Documento CEDES (117). Buenos Aires, Serie Educación Superior. 
Mignone, E. (1995). "Educación en los 90: el desafío de la calidad, la pertinencia, la eficacia y la equidad". Boletín de la Academia Nacional de Educación (19), pp. 4 -15. Argentina. Academia Nacional de Educación.

Mollis, M. (1994). Crisis, calidad y evaluación de las universidades: tres temas para el debate. En Krotsch, P., Puiggros A. (Comp.). Universidades y Evaluación: estado de la discusión. Buenos Aires. Editorial Aike.

Mundet, E. (2002). "El artículo 43 de la Ley 24.521: pautas para la interpretación y aplicación de la norma". Mimeo. Buenos Aires, Secretaría de Políticas Universitarias.

Recibido: 23/09/2010

Aceptado: 26/03/ 2012 